\title{
Correlation of Swallowing Function With Bilateral Diaphragmatic Movement in Hemiplegic Stroke Patients
}

\author{
Ra Yu Yun, MD, Ho Eun Park, MD, Ji Won Hong, MD, Yong Beom Shin, MD, PhD, Jin A Yoon, MD, PhD \\ Department of Rehabilitation Medicine, Pusan National University School of Medicine and Biomedical Research Institute, \\ Pusan National University Hospital, Busan, Korea
}

Objective To investigate difference in bilateral diaphragm movement of patients with tracheal aspiration according to post stroke residue severity and determine correlations of Penetration-Aspiration Scale (PAS), residue scale, and bilateral diaphragm movement.

Methods A total of 47 patients diagnosed with hemiplegic stroke were enrolled in this study. PAS, severity of valleculae, and pyriform sinus retention during videofluoroscopic swallowing study (VFSS) were assessed. Bilateral fluoroscopic diaphragm movements during spontaneous breathing and forced breathing were measured. Results Patients with tracheal aspiration (PAS $\geq 6)$ had significantly $(\mathrm{p}=0.035)$ lower ipsilateral diaphragm movement during spontaneous breathing. Post-swallow residue severity showed statistically significant $(\mathrm{p}=0.028)$ difference in patients with ipsilateral diaphragm movement during forced breathing. In linear regression analysis, PAS showed weak correlations with ipsilateral spontaneous diaphragm movement $(\mathrm{r}=0.397, \mathrm{p}=0.006)$, ipsilateral forced diaphragm movement $(\mathrm{r}=0.384, \mathrm{p}=0.008)$, and contralateral forced diaphragm movement $(\mathrm{r}=0.323$, $\mathrm{p}=0.027$ ). Weak correlation was also observed between post swallow residue severity and ipsilateral diaphragm movement during spontaneous breathing $(\mathrm{r}=0.331, \mathrm{p}=0.023)$ and forced breathing $(\mathrm{r}=0.343, \mathrm{p}=0.018)$.

Conclusion We confirmed the relationship between swallowing function and bilateral diaphragm movement in this study. The severity of dysphagia after hemiplegic stroke was correlated with bilateral diaphragm movement. Further longitudinal studies are needed to assess the effect of breathing exercise on post-stroke dysphagia.

Keywords Deglutition disorders, Diaphragm, Fluoroscopy, Respiration, Stroke

\footnotetext{
Received June 20, 2018; Accepted August 29, 2018

Corresponding author: Jin A Yoon

Department of Rehabilitation Medicine, Pusan National University School of Medicine and Biomedical Research Institute, Pusan National University Hospital, 179 Gudeok-ro, Seo-gu, Busan 49241, Korea. Tel: +82-51-240-7485, Fax: +82-51-247-7485, E-mail: yjk5289@naver.com

ORCID: Ra Yu Yun (http://orcid.org/0000-0003-3548-5784); Ho Eun Park (http://orcid.org/0000-0002-4254-0596); Ji Won Hong (http://orcid.org/00000002-0835-8213); Yong Beom Shin (http://orcid.org/0000-0001-5026-1696); Jin A Yoon (http://orcid.org/0000-0001-5762-0559).
}

(c) This is an open-access article distributed under the terms of the Creative Commons Attribution Non-Commercial License (http://creativecommons.org/ licenses/by-nc/4.0) which permits unrestricted noncommercial use, distribution, and reproduction in any medium, provided the original work is properly cited. Copyright @ 2019 by Korean Academy of Rehabilitation Medicine 


\section{INTRODUCTION}

The swallowing process needs coordinated movements of muscles of the mouth, pharynx, larynx, and esophagus [1]. These movements are all controlled by the central nervous system, particularly the cerebral cortex, medulla oblongata, and cranial nerve nuclei $[2,3]$. Dysphagia is common in stroke patients. The average incidence of dysphagia in stroke patients is approximately $40 \%$ [4].

Stroke patients with breathing difficulty may also have swallowing difficulty because of brief apnea during swallowing. The incidence of aspiration pneumonia is frequently associated with dysphagia [5-11]. Moreover, patients with decreased diaphragmatic movements may have high risk of aspiration pneumonia due to their insufficient capacity to induce effective coughing reflex [1214].

Given the importance of respiratory complications and dysphagia in patients' quality of life, we decided to assess the correlation between severity of post-stroke dysphagia and breathing difficulty. The National Institutes of Health Stroke Scale (NIHSS) is the most commonly used scale to evaluate stroke patients [15]. Modified Rankin Scale (mRS) is commonly used to measure the degree of disability or dependence of daily life in stroke patients. These scales are associated with patient's prognosis and quality of life $[16,17]$. Therefore, we examined difference in functional assessment battery according to swallowing function.

The objective of this study was to investigate differences in bilateral diaphragm movement for patients with tracheal aspiration according to post-stroke residue severity and determine correlations of Penetration Aspiration Scale (PAS), residue scale, and bilateral diaphragm movement.

\section{MATERIALS AND METHODS}

This was a prospective cross-sectional study to assess inpatients diagnosed with hemorrhagic or ischemic stroke who were referred to the rehabilitation department of a university hospital. All eligible patients were recruited from March 2017 to February 2018 at the time of evaluation. Inclusion criteria were: first-time subacute ( $<3$ months) stroke, age $\geq 19$ years at stroke onset, and corresponding lesion on computed tomography or mag- netic resonance imaging showing a single hemisphere lesion. Patients with histories of systemic illness or medication usage leading to neuropathy, loss of consciousness or lack of cooperative behavior, with sensory or global aphasia, having Mini-Mental State Examination (MMSE) score of less than 20 points, or having chest deformity were excluded from this study. All subjects provided written informed consent before inclusion in this study. Approval of this study was obtained from the Institutional Review Board of Pusan National University Hospital (No. H-1809-019-071).

\section{Evaluation of post-stroke dysphagia}

Videofluoroscopic swallowing study (VFSS) was performed to evaluate patients' swallowing function using Toshiba Ultimax-i DREX-UI80 (Toshiba America Medical System, Inc., Tustin, CA, USA) with digital storage of high-resolution images at a rate of 15 frames per second. Patients were examined in upright seated and lateral position. We use different quantities and consistencies of barium-impregnated bolus: 1, 3, 10, $20 \mathrm{~mL}$ of thin liquid and $3 \mathrm{~mL}$ of puree, semisolid, or solid. When fatal aspiration was observed, the examination was stopped.

The PAS was used to assess and grade the depth of airway invasion during VFSS [18]. PAS is designed to distinguish the depth of airway invasion in eight grades (grades 1 to 8) depending on the degree of invasion and aspiration and patient's response at the time of swallowing. In grade 1, there was normal swallowing without invasion or aspiration. Grade 8 indicated silent aspiration. In PAS, grade 6 or more means tracheal aspiration. Thus, we divided patients into two groups according to PAS grade: grades 1-5, patients without aspiration; and grades 6-8, patients with aspiration.

Pharyngeal residue is known to increase the risk of aspiration. In this study, four classification methods were used to classify residues in the valleculae or piriform sinuses during VFSS [19]: 0, absence of residue; 1 , mild residue, $25 \%$ of the height of the anatomical structure; 2 , moderate residue, $25 \%-50 \%$ of the height of the anatomical structure; and 3 , severe residue, more than $50 \%$ of the height of the anatomical structure.

\section{Diaphragm measurements by fluoroscopy}

In this study, bilateral diaphragmatic movements and swallowing test were evaluated simultaneously through 
fluoroscopy using Toshiba Ultimax-i DREX-UI80. Diaphragmatic movement was evaluated in supine position. It was measured by the highest point and the lowest point reached by the diaphragmatic cupula at excursion and incursion in both hemi-diaphragms during spontaneous breathing and forced breathing, respectively. A radiographic ruler was placed vertically behind the back of the patient and excursions of each hemidiaphragm apex were measured [20]. Each breathing cycle was performed more than five times with two rehabilitation physicians and the mean of 10 values was recorded. All evaluations and scoring were performed by two rehabilitation physicians. Final confirmations were performed by the corresponding author. Normal diaphragm movement during spontaneous breaching and forced breathing were defined as $1.8 \pm 0.3$ and $7.0 \pm 0.6 \mathrm{~cm}$, respectively, for males and $1.6 \pm 0.3$ and $5.7 \pm 1.0 \mathrm{~cm}$, respectively, for females [21].

\section{Functional assessment battery}

The mRS was used to assess patients' disabilities and the NIHSS was used to assess patients' severity of stroke attacks. mRS and NIHSS were assessed during swallowing and diaphragm function evaluation.

\section{Phrenic nerve conduction}

Phrenic nerve conduction was done to exclude patients with peripheral phrenic nerve palsy. Amplitude values at inspiration and expiration are different even in a normal person. Thus, the examination was done at the end of a breath cycle. Delayed latency was considered for latency greater than $8.0 \mathrm{~ms}$ and decreased amplitude for an amplitude less than $0.33 \mathrm{mV}$ [22].

\section{Statistical analysis}

The Mann-Whitney U test was used to investigate and compare dysphagia diaphragmatic movement, mRS, and NIHSS between the two groups divided by PAS grade. The Kruskal-Wallis test was used to investigate and compare diaphragmatic movement, mRS, and NIHSS among four groups divided by the amount of residue. We used MannWhitney U test with post hoc testing. Spearman correlation analysis and linear regression analysis were used to confirm correlations between swallowing function and diaphragmatic movement. p-values less than 0.05 were considered to indicate statistical significance. All statistical tests were conducted using SPSS Software version
22.0 (IBM SPSS, Armonk, NY, USA).

\section{RESULTS}

Initially, 60 patients diagnosed with stroke and requested for dysphagia evaluation were enrolled. Finally, 47 hemiplegic stroke patients (28 men and 19 women; age, $66.4 \pm 12.8$ years) satisfied our inclusion criteria. They

Table 1. Demographic and clinical data of patients

\begin{tabular}{|c|c|}
\hline Variable & Value \\
\hline \multicolumn{2}{|l|}{ Sex } \\
\hline Male & $28(59.6)$ \\
\hline Female & $19(40.4)$ \\
\hline Age (yr) & $66.4 \pm 12.8$ \\
\hline Onset (day) & $12.77 \pm 12.07$ \\
\hline $\mathrm{DM}$ & $10(21.3)$ \\
\hline \multicolumn{2}{|l|}{ Stroke type } \\
\hline Ischemic & $45(95.7)$ \\
\hline Hemorrhagic & $2(4.3)$ \\
\hline \multicolumn{2}{|l|}{ Affected level } \\
\hline Cortical & $8(17.0)$ \\
\hline Subcortical & $14(29.8)$ \\
\hline Brainstem & $4(8.5)$ \\
\hline Multiple & $21(44.7)$ \\
\hline \multicolumn{2}{|c|}{ Diaphragm movement } \\
\hline Normal & $4(8.5)$ \\
\hline Abnormal & $43(91.5)$ \\
\hline \multicolumn{2}{|l|}{ PAS } \\
\hline 0 & $0(0)$ \\
\hline 1 & $9(19.1)$ \\
\hline 2 & $5(10.6)$ \\
\hline 3 & $3(6.4)$ \\
\hline 4 & $4(8.5)$ \\
\hline 5 & $3(6.4)$ \\
\hline 6 & $3(6.4)$ \\
\hline 7 & $16(34.0)$ \\
\hline 8 & $4(8.5)$ \\
\hline \multicolumn{2}{|l|}{ Residue } \\
\hline 0 & $13(27.7)$ \\
\hline 1 & $12(25.5)$ \\
\hline 2 & $13(27.7)$ \\
\hline 3 & $9(19.1)$ \\
\hline
\end{tabular}

Values are presented as number (\%) or mean \pm standard deviation.

DM, diabetes mellitus. 
were enrolled in the study. The period from onset to enrollment was $12.77 \pm 12.07$ days. Four persons were within two standard deviations from normal diaphragm movement. There were 45 cases of ischemic stroke and 2 cases of hemorrhagic stroke. Of these 47 cases, 21 had right hemispheric stroke and 26 had left hemispheric stroke. Affected levels were as follows: cortical in 8, subcortical in 14, brainstem in 4, and multiple level in 21 cases (Table 1). Peripheral nerve lesion was not identified on phrenic nerve conduction.

Patients with tracheal aspiration (PAS $\geq 6$ ) had significantly $(\mathrm{p}=0.035)$ lower ipsilateral diaphragm movement during spontaneous breathing. Post-swallow residue severity showed statistically significant $(\mathrm{p}=0.028)$ difference in patients with ipsilateral diaphragm movement during forced breathing (Table 2). Post hoc testing with the Mann-Whitney $U$ test also showed significant difference between groups 0 and $3(\mathrm{p}=0.007)$, groups 1 and 3 $(\mathrm{p}=0.006)$, and groups 2 and $3(\mathrm{p}=0.030)$.

In linear regression analysis, PAS showed weak correlations with ipsilateral spontaneous diaphragm movement ( $\mathrm{r}=0.397, \mathrm{p}=0.006)$, ipsilateral forced diaphragm movement $(\mathrm{r}=0.384, \mathrm{p}=0.008)$, and contralateral forced diaphragm movement $(\mathrm{r}=0.323, \mathrm{p}=0.027)$. Weak correlation was also observed between post swallow residue severity and ipsilateral diaphragm movement during spontaneous breathing $(\mathrm{r}=0.343, \mathrm{p}=0.018)$ and forced breathing $(\mathrm{r}=0.331, \mathrm{p}=0.023)$ (Table 3).

After dividing patients with or without tracheal aspiration based on PAS grade, no significant difference in $\mathrm{mRS}$ $(p=1.0)$ or NIHSS ( $p=0.848)$ was observed between the two groups. There was no significant difference in mRS $(\mathrm{p}=0.773)$ or NIHSS $(\mathrm{p}=0.576)$ among the four groups divided based on the severity of residue either (Table 4).

\section{DISCUSSION}

In this study, we demonstrated that the extent of diaphragmatic movements in the ipsilateral side during forced or spontaneous breathing and in the contralateral side during forced breathing via fluoroscopic examination was correlated with patients' swallowing function. Several studies have reported that stroke can affect breathing, swallowing, and motor impairment [23]. However, to the best of our knowledge, no study has assessed swallowing functions with bilateral diaphragmatic movements through fluoroscopy in stroke patients. In this study, the diaphragmatic movement test by fluo-

Table 2. Difference in diaphragmatic movements between groups divided by swallowing function (PAS and amount of residue)

\begin{tabular}{lccccccccc}
\hline & \multicolumn{4}{c}{ PAS } & & \multicolumn{4}{c}{ Amount of residue } \\
\cline { 2 - 6 } \cline { 7 - 10 } & $\mathbf{5}$ & $\mathbf{5}$ & p-value & $\mathbf{0}$ & $\mathbf{1}$ & $\mathbf{2}$ & $\mathbf{3}$ & p-value \\
\hline Ipsilateral spontaneous & $1.91 \pm 0.69$ & $1.47 \pm 0.56$ & $0.035^{*}$ & & $1.98 \pm 0.75$ & $1.72 \pm 0.70$ & $3.65 \pm 1.18$ & $3.28 \pm 2.01$ & 0.200 \\
Ipsilateral forced & $3.81 \pm 1.66$ & $2.98 \pm 1.33$ & 0.133 & & $1.63 \pm 0.64$ & $1.62 \pm 0.64$ & $3.46 \pm 1.57$ & $3.19 \pm 1.28$ & $0.023^{*}$ \\
Contralateral spontaneous & $1.78 \pm 0.63$ & $1.63 \pm 0.62$ & 0.404 & & $1.73 \pm 0.48$ & $1.88 \pm 0.53$ & $4.04 \pm 1.83$ & $3.34 \pm 1.40$ & 0.772 \\
Contralateral forced & $3.61 \pm 1.68$ & $2.87 \pm 1.18$ & 0.123 & & $1.31 \pm 0.67$ & $1.62 \pm 0.66$ & $2.11 \pm 0.89$ & $3.17 \pm 1.20$ & 0.958 \\
\hline
\end{tabular}

Values are presented as mean \pm standard deviation.

PAS, Penetration Aspiration Scale.

${ }^{*} \mathrm{p}<0.05$.

Table 3. Correlation between swallowing function (PAS and amount of residue) and diaphragmatic movement

\begin{tabular}{lcc}
\hline & r & p-value \\
\hline PAS and ipsilateral spontaneous diaphragm movement & 0.397 & $0.006^{*}$ \\
\hline PAS and ipsilateral forced diaphragm movement & 0.384 & $0.008^{*}$ \\
PAS and contralateral forced diaphragm movement & 0.323 & $0.027^{*}$ \\
Amount of residue and ipsilateral spontaneous diaphragm movement & 0.343 & $0.018^{*}$ \\
Amount of residue and ipsilateral forced diaphragm movement & 0.331 & $0.023^{*}$ \\
\hline
\end{tabular}

PAS, Penetration Aspiration Scale.

${ }^{*} \mathrm{p}<0.05$. 
Table 4. Difference in functional ability (MMT, mRS, NIHSS) between groups divided by swallowing function (PAS and amount of residue)

\begin{tabular}{|c|c|c|c|c|c|c|c|c|}
\hline & \multicolumn{3}{|c|}{ PAS } & \multicolumn{5}{|c|}{ Amount of residue } \\
\hline & $\leq 5$ & $\geq 6$ & p-value & $\mathbf{0}$ & 1 & 2 & 3 & p-value \\
\hline $\mathrm{mRS}$ & $4.13 \pm 0.80$ & $4.09 \pm 0.90$ & 1.0 & $3.92 \pm 0.95$ & $4.25 \pm 0.62$ & $4.08 \pm 0.86$ & $4.22 \pm 0.97$ & 0.773 \\
\hline NIHSS & $10.17 \pm 5.90$ & $10.30 \pm 4.39$ & 0.848 & $9.54 \pm 6.17$ & $12 \pm 4.75$ & $9.38 \pm 4.77$ & $10.11 \pm 4.94$ & 0.576 \\
\hline
\end{tabular}

Values are presented as mean \pm standard deviation.

mRS, modified Rankin scale; NIHSS, National Institutes of Health Stroke Scale; PAS, Penetration Aspiration Scale.

roscopy was performed simultaneously with VFSS for a short period of time. This is advantageous to observe the movement of the diaphragm objectively rather by ultrasonography. Our results revealed that forced breathing and swallowing were related to each other. Diaphragmatic movement during deep breathing may be considered similar to forced vital capacity. Forced vital capacity refers to air volume that can be exhaled when deep exhalation occurs. Compared with spontaneous breathing, forced breathing was associated with swallowing function more. The difference in diaphragmatic movements during spontaneous breathing was very small which might limit patient comparisons. Such difference might be due to large difference in forced breathing.

Stroke may disrupt breathing in many ways. It may influence respiration rhythm, cause problems in the respiratory pathway from the brain, and affect diaphragmatic movement. In the respiratory process, the diaphragm is the most important organ. Diaphragmatic movement is regulated by several pathways. Two major descending pathways are the corticospinal pathway from the cortex to respiratory motor neurons known to be responsible for voluntary breathing [24] and the bulbospinal pathway from the medulla to the respiratory motor neurons that is associated with the control of automatic breathing [25]. Moreover, a putative connection between the motor cortex and pontomedullary respiratory centers exists [26]. Because of these various control pathways, bilateral diaphragmatic movements in stroke patients may vary. In addition, the correlation between breathing and swallowing processes is clearly demonstrated by shared muscle components and anatomical structures. Breathing shares many muscles involved in swallowing $[27,28]$. There is approximately 1 second of apnea during swallowing [29]. Patients with poor pulmonary function have difficulty holding their breath which in turn affects their swallowing function. Moreover, during swallowing, non-spontaneous respiration should be controlled. This is done by the swallowing center. If damage occurs, this process becomes problematic [30]. Therefore, several previous studies have attempted to confirm the association of swallowing function with respiratory function.

Fregosi and Fuller [27] have measured the diaphragmatic movement in stroke patients with or without swallowing difficulty and healthy control group by M-mode ultrasonography while patients perform quiet breathing, deep breathing, and voluntary coughing. The phrenic nerve has a corticospinal projection. Hence, when comparing relationships between swallowing function and respiration, ipsilateral and contralateral sides of the diaphragm should be evaluated and their values showed be compared with swallowing function rather than only numerically comparing one side of diaphragmatic movement. However, in their study, only the diaphragm of the hemi-side was evaluated. Thus, we compared diaphragmatic movements on both sides. Fregosi and Fuller [27] used ultrasonography to evaluate diaphragmatic movement. The most popular method to evaluate diaphragmatic movement is ultrasonography. However, the quality of ultrasonography evaluation depends on technical skill of the operator. The gold standard for evaluating swallowing dysfunction is fluoroscopy. Hence, after VFSS, fluoroscopic diaphragm examination was done in the same laboratory.

In one study, nasal cannulae were used to determine differences in respiratory air flow during swallowing between normal and stroke patients. However, there was no statistically significant difference in apnea time. In addition, there is no evidence linking swallowing respiratory characteristics to aspiration [31]. However, mRS or NIHSS, the most basic functional assessment of stroke patients, was not significantly associated with dysphagia severity. The present study suggests that mRS or NIHSS cannot assess patient's impairment in a multidisciplinary 
manner. This is probably because neither tool has a domain for dysphagia evaluation. Because these scales do not predict the reduction in quality of life (QOL) due to dysphagia, a common domain to assess activities of daily living, QOL depression due to dysphagia needs to be assessed using America Speech-Language Hearing Association National Outcome Measurement System swallowing scale.

This study has some limitations. First, because patient coordination is important to perform the diaphragmatic movement test and VFSS, all patients with an MMSE of 20 or less were excluded from enrollment. Hence, the number of subjects enrolled was very small. A similar study with a larger sample size will be needed to generalize results of this study. Second, most stroke patients included in our study were ischemic stroke patients and the distribution of patients was biased to one side. In addition, the proportion of the affected site of stroke in patients was not evenly distributed. In the future, it will be necessary not only to increase the number of patients, but also to make the distribution of patient groups more uniform so that the affected side of stroke does not become a bias in the study. Third, diaphragm movement of most patients was deviated from the normal range. This is due to the fact that no paper has published the normal range by measuring diaphragm movement using fluoroscopy. Thus, we compared diaphragm movement with the normal value using ultrasonography. In addition, because there were too many patients out of the normal range, there was a limit in additional analysis to result in statistically significant results except for these patients.

In conclusion, this study evaluated swallowing function and diaphragmatic movement through fluoroscopic examination and confirmed the relationship between swallowing function and bilateral diaphragmatic movement. Deep breathing exercise in addition to dysphagia therapy to enhance diaphragmatic movement could be administered to patients with swallowing problems and patients who cannot breathe deeply. Further longitudinal studies are needed to assess the effect of breathing exercise on post-stroke dysphagia.

\section{CONFLICT OF INTEREST}

No potential conflict of interest relevant to this article was reported.

\section{ACKNOWLEDGMENTS}

This work was supported by clinical research grant from Pusan National University Hospital in 2018.

\section{AUTHOR CONTRIBUTION}

Conceptualization: Yun RY, Yoon JA. Methodology: Shin YB. Formal analysis: Yun RY. Project administration: Yun RY, Yoon JA. Visualization: Hong JW, Park HE. Writing original draft: Yun RY. Writing - review and editing: Yoon JA, Shin YB. Approval of final manuscript: all authors.

\section{REFERENCES}

1. Jeon WH, Park GW, Lee JH, Jeong HJ, Sim YJ. Association between location of brain lesion and clinical factors and findings of videofluoroscopic swallowing study in subacute stroke patients. Brain Neurorehabil 2004;7:54-60.

2. Cunningham ET, Donner MW, Jones B, Point SM. Anatomical and physiological overview. In: Jones B, Donner MW, editors. Normal and abnormal swallowing. New York: Springer; 1991. p. 7-32.

3. Miller AJ. Characteristics of the swallowing reflex induced by peripheral nerve and brain stem stimulation. Exp Neurol 1972;34:210-22.

4. National Collaborating Centre for Chronic Conditions. Stroke: national clinical guideline for diagnosis and initial management of acute stroke and Transient Ischaemic Attack (TIA). London: Royal College of Physicians; 2008.

5. Hannawi Y, Hannawi B, Rao CP, Suarez JI, Bershad EM. Stroke-associated pneumonia: major advances and obstacles. Cerebrovasc Dis 2013;35:430-43.

6. Finlayson O, Kapral M, Hall R, Asllani E, Selchen D, Saposnik G, et al. Risk factors, inpatient care, and outcomes of pneumonia after ischemic stroke. Neurology 2011;77:1338-45.

7. Sellars C, Bowie L, Bagg J, Sweeney MP, Miller H, Tilston J, et al. Risk factors for chest infection in acute stroke: a prospective cohort study. Stroke 2007;38: 2284-91.

8. Chumbler NR, Williams LS, Wells CK, Lo AC, Nadeau S, Peixoto AJ, et al. Derivation and validation of a clinical system for predicting pneumonia in acute stroke. 
Neuroepidemiology 2010;34:193-9.

9. Lakshminarayan K, Tsai AW, Tong X, Vazquez G, Peacock JM, George MG, et al. Utility of dysphagia screening results in predicting poststroke pneumonia. Stroke 2010;41:2849-54.

10. Smithard DG, O’Neill PA, Parks C, Morris J. Complications and outcome after acute stroke. Does dysphagia matter? Stroke 1996;27:1200-4.

11. Mo SJ, Jeong HJ, Han YH, Hwang K, Choi JK. Association of brain lesions and videofluoroscopic dysphagia scale parameters on patients with acute cerebral infarctions. Ann Rehabil Med 2018;42:560-8.

12. Zhou Z, Vincent F, Salle JY, Antonini MT, Aliamus V, Daviet JC. Acute stroke phase voluntary cough and correlation with maximum phonation time. Am J Phys Med Rehabil 2012;91:494-500.

13. Harraf F, Ward K, Man W, Rafferty G, Mills K, Polkey $\mathrm{M}$, et al. Transcranial magnetic stimulation study of expiratory muscle weakness in acute ischemic stroke. Neurology 2008;71:2000-7.

14. Ward K, Seymour J, Steier J, Jolley CJ, Polkey MI, Kalra $\mathrm{L}$, et al. Acute ischaemic hemispheric stroke is associated with impairment of reflex in addition to voluntary cough. Eur Respir J 2010;36:1383-90.

15. Saito J, Koyama T, Domen K. Long-term outcomes of FIM motor items predicted from acute stage NIHSS of patients with middle cerebral artery infarct. Ann Rehabil Med 2018;42:670-81.

16. Ali M, Fulton R, Quinn T, Brady M; VISTA Collaboration. How well do standard stroke outcome measures reflect quality of life? A retrospective analysis of clinical trial data. Stroke 2013;44:3161-5.

17. Rangaraju S, Haussen D, Nogueira RG, Nahab F, Frankel M. Comparison of 3-month stroke disability and quality of life across Modified Rankin Scale categories. Interv Neurol 2017;6:36-41.

18. Rosenbek JC, Robbins JA, Roecker EB, Coyle JL, Wood JL. A penetration-aspiration scale. Dysphagia 1996;11: 93-8.

19. Eisenhuber E, Schima W, Schober E, Pokieser P, Stadler A, Scharitzer M, et al. Videofluoroscopic assessment of patients with dysphagia: pharyngeal re- tention is a predictive factor for aspiration. AJR Am J Roentgenol 2002;178:393-8.

20. Houston JG, Fleet M, Cowan MD, McMillan NC. Comparison of ultrasound with fluoroscopy in the assessment of suspected hemidiaphragmatic movement abnormality. Clin Radiol 1995;50:95-8.

21. Boussuges A, Gole Y, Blanc P. Diaphragmatic motion studied by m-mode ultrasonography: methods, reproducibility, and normal values. Chest 2009;135:391400.

22. Resman-Gaspersc A, Podnar S. Phrenic nerve conduction studies: technical aspects and normative data. Muscle Nerve 2008;37:36-41.

23. Voyvoda N, Yucel C, Karatas G, Oguzulgen I, Oktar S. An evaluation of diaphragmatic movements in hemiplegic patients. Br J Radiol 2012;85:411-4.

24. Mitchell RA, Berger AJ. Neural regulation of respiration. Am Rev Respir Dis 1975;111:206-24.

25. Nathan PW. The descending respiratory pathway in man. J Neurol Neurosurg Psychiatry 1963;26:487-99.

26. Abdala AP, Rybak IA, Smith JC, Zoccal DB, Machado $\mathrm{BH}$, St-John WM, et al. Multiple pontomedullary mechanisms of respiratory rhythmogenesis. Respir Physiol Neurobiol 2009;168:19-25.

27. Fregosi RF, Fuller DD. Respiratory-related control of extrinsic tongue muscle activity. Respir Physiol 1997;110:295-306.

28. van Lunteren E, Dick TE. Intrinsic properties of pharyngeal and diaphragmatic respiratory motoneurons and muscles. J Appl Physiol (1985) 1992;73:787-800.

29. Martin BJ, Logemann JA, Shaker R, Dodds WJ. Coordination between respiration and swallowing: respiratory phase relationships and temporal integration. J Appl Physiol (1985) 1994;76:714-23.

30. Selley WG, Flack FC, Ellis RE, Brooks WA. Respiratory patterns associated with swallowing. Part 2. Neurologically impaired dysphagic patients. Age Ageing 1989;18:173-6.

31. Leslie P, Drinnan MJ, Ford GA, Wilson JA. Swallow respiration patterns in dysphagic patients following acute stroke. Dysphagia 2002;17:202-7. 